\title{
What can we Learn from Oscillation Studies about Irradiance and Radius Changes?
}

\author{
By D OUGLAS G OUGH
}

Institute of Astronomy and Department of Applied Mathematics and Theoretical Physics, University of Cambridge, UK and Joint Institute for Laboratory Astrophysics, University of Colorado and National Institute of Standards and Technology, Boulder, CO 80303, USA

Dynamical and thermal modulation of the internal structure of the Sun can be manifest at the surface as changes in irradiance and radius. The relative magnitudes of these changes could provide a diagnostic of at least the location of the primary modulation, if only the mechanism were known. Variations of the frequencies of solar oscillations offer additional potentially valuable diagnostics, but unfortunately at present the mechanisms causing those variations and their relation to the structural and irradiance changes are not yet understood. In this lecture I shall review some of the theoretical conjectures that have been put forward to explain the observations.

\section{Introduction}

Associated with the solar cycle are variations in irradiance and in the apparent radius of the photosphere. How do these arise? I do not know the answer. However, recent helioseismic data have enabled us to eliminate some notions. In this review I shall try to explain how. To set the scene, let me first recall Kuhn's presentation at this colloquium, in which he analysed the latitudinal variation of the brightness of the solar surface and deduced that the luminosity of the Sun also varies with the cycle; it is almost in step with the irradiance variation and has essentially the same amplitude. This is an important result, for it implies that the spherically averaged thermal structure of the Sun varies with the cycle. There is also an aspherical component, associated with which must be large-scale material circulation, advecting heat, magnetic fields and angular momentum. Without an understanding of these processes we cannot understand the solar cycle, and without some means of measuring them, we cannnot test our theories adequately. Therefore I shall discuss also what has recently been learnt about variations of the Sun's angular velocity.

\section{The luminosity and radius variations}

Even without a precise model of solar variation, some information can be gleaned from the ratio $W$ of the relative variations in the photospheric radius $R$ and luminosity $L: W=\Delta \ln R / \Delta \ln L$. This was a quantity of some interest a decade or so ago, for its magnitude provides some indication of where in the Sun the process directly responsible for changing the structure is located. On the basis of a few particular calculations, it has been conjectured (Gough 1981) that if the radius and luminosity variations have the same origin, at some radius $r_{0}$, then on the whole $W$ is a positive decreasing function of $r_{0}$, and virtually vanishes as $r_{0} \rightarrow R$. In particular, $W \simeq 0.5$ when $r_{0}=0$, and $W \simeq 0.2$ when the perturbing process is situated near the base of the convection zone; a modification to the upper superadiabatic convective boundary layer yields a very much smaller value: $W \lesssim 10^{-3}$.

The reason for this dependence on radius is not difficult to appreciate. First, let us 
remind ourselves that the characteristic internal thermal readjustment time for the convection zone is given by $\tau_{\mathrm{a}}=\int_{r_{\mathrm{c}}}^{R} w^{-1} d r \simeq 1 \mathrm{y}$, where $w$ is the rms vertical velocity of the convective motion and $r=r_{\mathrm{c}}$ locates the base of the convection zone, whereas the thermal cooling time for the zone as a whole is $\tau_{\mathrm{c}} \simeq 10^{5} \mathrm{y}$, and, because it is much longer than $\tau_{\mathrm{a}}$, is determined only by the heat content of the zone and the radiative properties of the photosphere (Gough 1981; Spruit 1990). Thus, on a timescale of 11y, the convection zone can be considered to be almost in internal thermal balance, providing a large reservoir to supply or absorb heat associated with any perturbation to the luminosity without a significant concomitant change in its hydrostatic stratification. Any modification to the thermal impedance of the surface layers generally changes both $L$ and the stratification of the superadiabatic boundary layer by similar amounts, but does not modify the adiabat beneath. Consequently if $b$ is the characteristic thickness of the boundary layer, $\delta \ln L$ and $\delta \ln b$ are of the same order, and $|W|=\mathrm{O}(b / r) \ll 1$. If, on the other hand, the perturbation is beneath the convection zone, the reaction of the zone involves an overall hydrostatic change, which typically entails comparable changes in $\ln R$ and $\ln L$.

It is important to realize that this argument is specific to timescales $\tau$ satisfying $\tau_{\mathrm{a}} \ll$ $\tau \ll \tau_{\mathrm{c}}$. As $\tau$ increases towards $\tau_{\mathrm{c}}$ the thermal reservoir responds more, and $W$ increases; and once $\tau$ becomes comparable with the thermal diffusion time of the radiative interior, $\tau_{\mathrm{KH}} \simeq 3 \times 10^{7} \mathrm{y}$, the entire star responds. Däppen (1983) has shown how this trend can be studied. In particular, when $\tau \gg \tau_{\mathrm{KH}}$, increasing the efficacy of convection (whose immediate effect is to modify the stratification of the thin boundary layer) causes the entire star to shrink to a new state of thermal balance, and the value of $W$ becomes quite large and negative (cf. Ulrich 1986; Gough \& Novotny 1993). Now the luminosity is determined not solely by the thermal properties of the photosphere, but also by the augmented rates of the thermonuclear reactions under the increased weight of the more compact star.

The solar-cycle variation of irradiance (Willson \& Hudson 1988) is now well established (e.g. Kyle et al. 1994). However, positive reports of radius variations are controversial, partly because the observations are difficult to interpret. Brown (1987), for example, finds no significant signal; his upper bound implies $|W| \lesssim 5 \times 10^{-2}$, apparently implying that the thermal variation substantially modifies the structure of the Sun only in its outer layers. On the other hand, Delache et al. (1993) take the CERGA astrolobe data literally as measurements of $R$, from which one would deduce $W \simeq-0.3$. If correct, that implies the existence of a deeply seated process modifying the stratification of the Sun. However, it is difficult to reconcile so large a negative $W$ on an 11-year timescale with a single process. If the processes that regulate $R$ and $L$ were different, though presumably connected by some solar-cycle mechanism, then $W$ would no longer be a robust indicator of the whereabouts of those processes, even if they occurred in the same place.

\section{Lifting of p-mode degeneracy}

The publication by Duvall et al. (1986) of an even component to the frequencyaveraged splitting of p-mode multiplet frequencies, observed near sunspot maximum in winter 1981-82, demonstrated that the hydrostatic structure of the Sun was not spherically symmetric. The asphericity was a factor 10 greater than the oblateness caused by the centrifugal acceleration from the angular velocity $\Omega$ ( $\Omega$ is measured by the odd component of the splitting) and of the opposite sign; acoustically, the Sun was prolate.

In attempting to explain the phenomenon, Gough \& Thompson (1988) observed that the dependence of the splitting on $m / \Lambda$, where $m$ is azimuthal order and $\Lambda^{2}=\ell(\ell+1)$, $\ell$ being the degree of the mode, though not well resolved, implied a latitudinal variation 


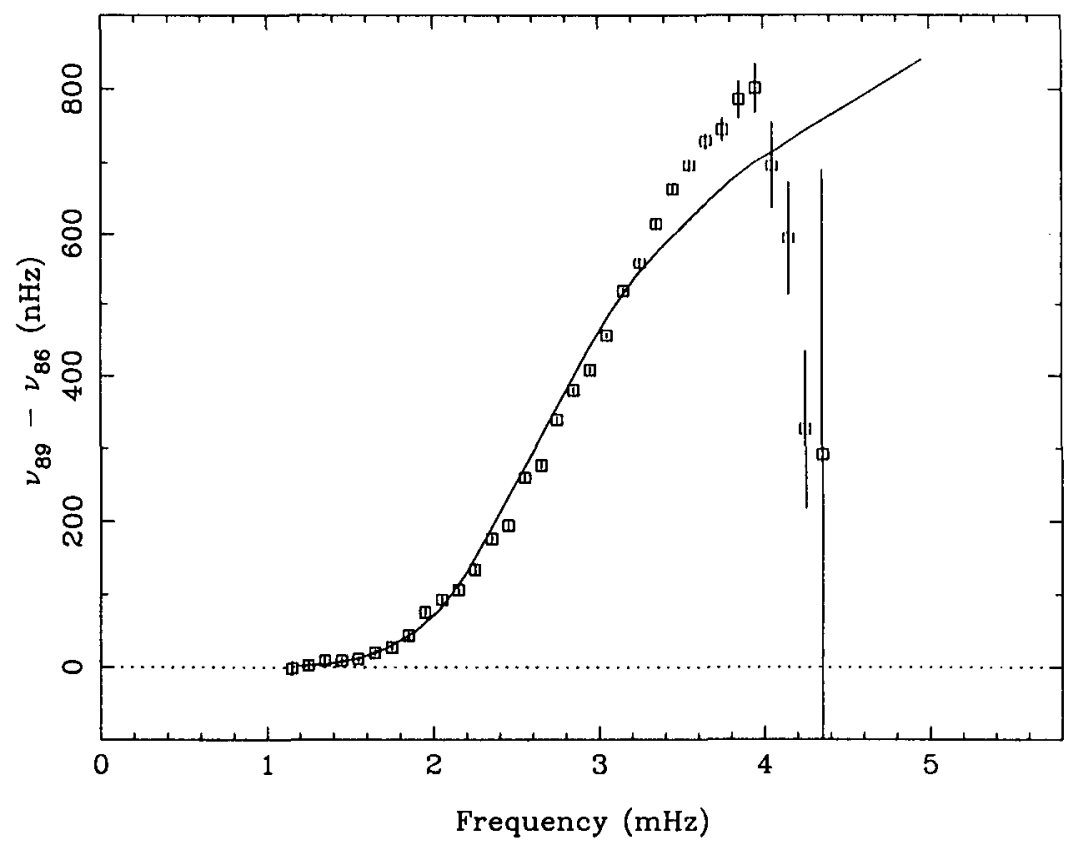

Figure 1. Frequency changes $\nu_{89}-\nu_{86}$ from 1986 to 1989 measured at the Big Bear Solar Observatory, averaged in $100 \mu \mathrm{Hz}$ bins. The continuous line is proportional to the inertia of low-degree modes, normalized at a height of $430 \mathrm{~km}$ above the photosphere (after Woodard \& Libbrecht 1993b).

of the hydrostatic stratification not dissimilar to that of the sunspots. They supposed that the frequency perturbations were due to magnetic activity in the form of fibril fields, which augmented the wave propagation speed and thereby increased the frequencies of the acoustic modes. The variation from pole to equator of fibril intensity required to reproduce the observations was not obviously at variance with observation, so the conjecture was not implausible. By considering the dependence of the degeneracy splitting on $\Lambda$, the variation of the fibril perturbation with depth can in principle be ascertained. Those early data were quite noisy. Nonetheless, they indicated that the perturbation was somewhat concentrated near the surface of the Sun, but that it was not completely confined there. Indeed, by fitting the data to a power law in $\Lambda$, a dependence on depth $R-r$ of the form $(R-r)^{0.1}$ was inferred.

Subsequent observations showed that the frequency splitting diminished towards solar minimum. The data were considered by Kuhn (1988a, 1988b) who compared them with surface brightness temperature measurements (Kuhn et al. 1988). Kuhn pointed out that if one were to assume simply that there were a depth-independent (at least down to $r=0.95 R$ and possibly as deep as $r=0.2 R$ ) relative sound-speed variation of the same magnitude as that implied by the apparent surface temperature variation, with no other modification to the Sun's structure, then the oscillation data could be reproduced. As will become evident in the next section, it appears that that idea cannot describe what is actually the case, because, as with the latitudinal variation, the temporal acoustical changes are concentrated very near the surface of the Sun. However, the coincidence is striking, and it would be interesting to find out whether it is a signature of an important physical constraint, or is merely fortuitous. 


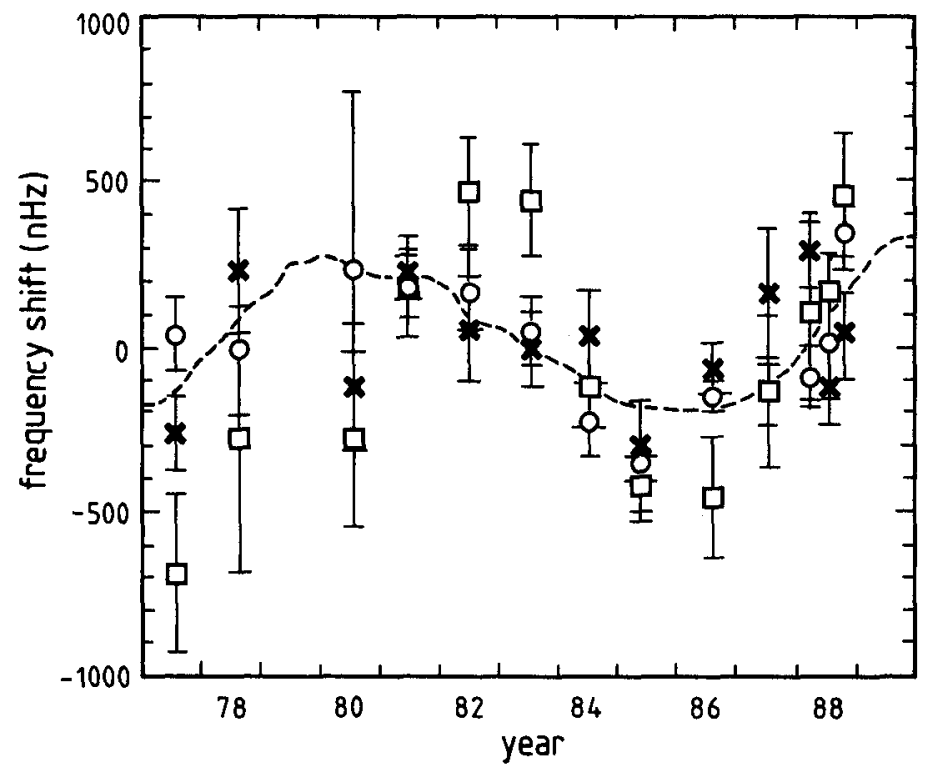

Figure 2. Frequency shifts of low-degree p modes, obtained from the whole-disk Doppler measurements from the Birmingham Solar Network (after Elsworth et al. 1990). The dashed line represents sunspot number, and is plotted on a scale chosen to fit the frequency shifts.

\section{Solar-cycle frequency variations}

A substantial step forward was made in 1990 with two announcements of temporal frequency changes. One was by Libbrecht \& Woodard (1990), who reported changes from 1986 to 1988 both in multiplet frequencies (the mean frequencies of modes of like order $n$ and degree $\ell$, averaged over all admissible values of $m$; they depend on the spherically averaged structure of the star) and in the even contribution to degeneracy splitting (expressed, essentially as also had Duvall, Harvey and Pomerantz and subsequent observers, as coefficients $\alpha_{i}$ in a Legendre series expansion in the variable $m / \Lambda$ ). For the first time, the frequency dependence of the data was presented, and it was shown that the changes in both the multiplet frequencies (Figure 1) and the even splitting coefficients $\alpha_{i}(\omega, \ell)$ were roughly inversely proportional to the inertia $I$ of the mode. The other announcement, by Elsworth et al. (1990), was of a variation over an 11-year interval of low-degree frequencies (averaged over $n$ ) of Doppler measurements in spatially integrated light (Figure 2 ), which demonstrated a connexion with the solar cycle. Like the luminosity, oscillation frequencies are greatest at solar maximum, the total variation being about $0.46 \mu \mathrm{Hz}$.

The importance of the $I^{-1}$ proportionality can be appreciated from the formula $\delta \nu \simeq$ $\nu^{-1} I^{-1} \int\left(\mathcal{K}-\nu^{2} \mathcal{I}\right) d V$ for the small difference between the cyclic frequencies $\nu$ of corresponding modes of two similar states of the Sun; the integrands $\mathcal{K}$ and $\mathcal{I}$ are sums of terms which are bilinear in the components, or their spatial derivatives, of the displacement eigenfunction $\xi$ of one of the states of the Sun (the reference), and which are linear in quantities defining the difference between the two states, and they are integrated over the volume $V$ of the reference state. The inertia $I$ is given by $\int \rho \xi \cdot \xi d V$, which is also bilinear in $\xi$, so the arbitrary amplitude of $\xi$ cancels. For convenience, suppose the eigenfunctions to be normalized such that the average of $|\xi|$ over the surface of the Sun is unity. Then 
it turns out that $I$ is a rapidly decreasing function of $\nu$ at low $\nu$; roughly, $I \propto \nu^{-\alpha}$ with $\alpha \simeq 7$, the precise value of $\alpha$ depending on where in the solar atmosphere $\xi$ is normalized. Thus, to within low powers of $\nu$, it is evident that $\delta \nu$ is approximately proportional to $I^{-1}$ if $\int \mathcal{K} d V$ and $\int \mathcal{I} d V$ are constants, independent of which mode is being considered; that is achieved if $\mathcal{K}$ and $\mathcal{I}$ differ from zero only in the very surface layers near where $\xi$ is normalized. Thus the data of Libbrecht and Woodard convincingly demonstrated that both the mean and the latitudinally varying change in the solar structure occurs predominantly in the outer layers. With this in mind, one could then demonstrate that the low-degree data were similarly explained; indeed, the frequency dependence of frequency differences between sunspot maximum and sunpot minimum determined by $P$. L. Pallé, C. Régulo and T. Roca Cortés, though noisy, were not inconsistent with the $I^{-1}$ scaling (Gough 1990a). I should point out that because $I$ is so rapidly varying a function of $\nu$ at low $\nu$, it is not easy to tell whether $\delta \nu$ is proportional strictly to $I^{-1}$ or to some low-degree polynomial in (or slowly varying function of) $\nu$ multiplied by $I^{-1}$. Indeed, one would expect the latter, the details depending on precisely in what manner the outer layers of the Sun vary. For example, it has been argued (Gough 1990b) that the relative frequency variation is likely to be of the form $\left(\nu^{2} I\right)^{-1} Q(\nu)$, where $Q$ is a low-degree polynomial.

\section{How can the frequency variations be explained?}

I have already mentioned that it has been suggested (Gough \& Thompson 1988) that a latitudinal variation of fibril magnetic field in the outer layers of the Sun is responsible for the degeneracy splitting. It is natural to adopt a unified conjecture, and to suppose that temporal variations in the same field are responsible for the solar-cycle changes too. This conjecture has been taken up by Goldreich et al. (1991), who discuss the influence on the oscillations in more detail. They also found that the magnitude of the field variation required is not obviously at variance with observation. Strong evidence for a unified cause of both temporal multiplet frequency variations and degeneracy splitting has been provided recently by Woodard et al. (1991), Woodard \& Libbrecht (1993a), and Backman \& Brown (1993), who demonstrate that there is a unique relationship between oscillation frequency changes and surface activity, irrespective of the characteristic timescale of the variation.

It is perhaps more natural to suppose that the frequency shifts result from a thermal variation modifying the sound speed, brought about, for example, by a modification to the efficacy of convection by a magnetic field. This has been discussed in various guises by Kuhn (1988a, 1988b, 1989a, 1989b) following ideas developed by Parker (1987). He basically postulates thermal disturbances rising from regions of magnetic activity at the base of the convection zone. The numerical experiment he discusses in these proceedings indicates that the relative temperature fluctuation increases near the top of the convection zone, and thereby modifies the sound speed predominantly in the outer layers of the Sun, as implied by Libbrecht and Woodard's data. However, ideas of this kind are difficult to reconcile with the observed luminosity variations. Indeed, Gough \& Thompson (1988) had already considered the possibility that such thermal disturbances are responsible for degeneracy splitting, as an alternative to the direct dynamical effect of a fibril magnetic field. They modified the efficacy of convection by changing the mixing-length parameter in a model of the solar envelope that matches onto an unperturbed radiative interior. Although it was possible to reproduce the splitting data, it required a radiative flux some 15 per cent smaller at the equator than at the poles. This is contradicted by the 
surface brightness measurements published by Kuhn et al. (1985, 1988); not only were the theoretical flux variations too large by a factor of about 50 , but they also had the wrong sign. A similar conclusion was drawn subsequently by Goldreich et al. (1991) and Balmforth et al. (1993) when considering solar cycle variations of multiplet frequencies.

The reason why purely thermal readjustments lead to the wrong sign of the frequency perturbation relative to the luminosity change is easy to explain (Goldreich et al. 1991; Balmforth et al. 1993). It is adequate to consider spherical perturbations, such as can result from increasing the efficacy of convection, which, on a timescale of $11 y$ diminishes the superadiabatic gradient $\Delta \nabla \equiv d \ln T / d \ln p-(\partial \ln T / \partial \ln p)_{\text {ad }}$ in the boundary layer without modifying the structure of the adiabatically stratified interior. (The entropy deep in the interior is essentially unchanged on timescales much less than $\tau_{\mathrm{c}}$, and therefore the pressure $p$, and consequently temperature $T$ and density $\rho$, are also unchanged, because a slight expansion or contraction of the outer layers hardly changes their weight.) The dominant change is to the gradient $d \ln T / d \ln p, \operatorname{since}(\partial \ln T / \partial \ln p)_{\text {ad }}$ is a thermodynamic quantity and is more robust; therefore the temperature of a given material layer in the vicinity of the photosphere increases. Gravity hardly varies through the thin outer layers, and pressure, which depends on the weight of the overlying material, is essentially a Lagrangian variable (being directly related to the weight, and therefore the mass of the overlying material). Thus, the photospheric temperature increases, and so does the radiative flux. Roughly speaking, the oscillation frequencies $\nu$ are proportional to $\left(\int c^{-1} d r\right)^{-1}$, which can be considered to be both proportional to sound speed $c \propto T^{1 / 2}$ and inversely proportional to the characteristic length scale $\lambda$ of the cavity within which the modes are confined. If one disregards the movement of the outer boundary of the cavity with respect to Lagrangian coordinates (which is a good first approximation), then, since the Lagrangian pressure perturbation $\delta p$ vanishes, $\delta \lambda / \lambda=-\delta \rho / \rho=\delta T / T$, whereas $\delta c / c=\frac{1}{2} \delta T / T$. Thus $\delta \nu / \nu=\delta c / c-\delta \lambda / \lambda=-\frac{1}{2} \delta T / T$. The relative increase in the cavity size resulting from heating the layer is twice the relative increase in sound speed, and consequently the frequencies of oscillation are reduced. To show that the magnitude of the frequency change is much too small it is hardly necessary to be subtle. It suffices to estimate $\delta \nu / \nu$ by $-\frac{1}{2} \int T^{-1} \delta T d \tau / \int d \tau$ where $d \tau=c^{-1} d r$. For low-degree modes the integrals are taken over the entire Sun, whose acoustical radius is $\int d \tau \simeq 3500 \mathrm{~s}$. Taking $\delta T / T$ to decline linearly with respect to $\tau$, from its photospheric value of $2.5 \times 10^{-4}$ implied by a $10^{-3}$ relative variation in $L$ to zero near the bottom of the boundary layer, at say where hydrogen is 50 per cent ionized (at an acoustical depth of about $200 \mathrm{~s}$ ), therefore leads to a frequency variation of $0.011 \mu \mathrm{Hz}$ for a $3 \mathrm{mHz}$ mode. This is smaller than the value reported by Elsworth et al. (1990) by a factor of more than 40 .

Because this result deviates by so much from observation it seems inevitable that a very different mechanism must be responsible for the frequency variations. Neglected Reynolds stresses or slow large-scale circulation in the convection zone is hardly likely to alter the argument materially. Moreover, Balmforth et al. (1993), who also performed a time-dependent calculation when perturbing the star, have confirmed that nonadiabatic processes also play only a minor role. Aside possibly from horizontal inhomogeneity, the direct influence of Lorentz forces on the acoustic oscillations appears to be the most likely remaining candidate for causing the variation. This could be of the form of a fibril field in and beneath the photosphere, as I have already discussed. Alternatively, it has been suggested that instead it might be a relatively smooth horizontal field in the chromosphere (Campbell \& Roberts (1983); Evans \& Roberts (1990, 1991); Wright \& Thompson 1992). Whatever is the case, it is unfortunate that there is no robust theory to link the variations of the magnetic field to those in irradiance. 


\section{Variations in the angular velocity}

One expects horizontal variations in structure to be intimately linked with material circulation and consequent redistribution of angular momentum. Therefore angular velocity variations represent an integral aspect of solar variability. The original inversion of rotational splitting of sectoral modes (Duvall et al. 1984) revealed a radial variation of angular velocity $\Omega$ in the vicinity of the equatorial plane that immediately suggested temporal variation (Gough 1985). Goode et al. (1991) claimed that subsequent helioseismic data sets indeed provided direct evidence for such a variation, particularly in the radiative interior. However, the significance of the claim has since been brought into doubt (Schou 1991; Goode \& Dziembowski 1993; Gough \& Stark 1993a; Woodard \& Libbrecht 1993a).

Significance of inferences from helioseismic data is difficult to assess, because the reliability of the means by which uncertainties in the data are estimated is hard to judge. Of special concern are systematic errors. When looking for temporal trends it is always safer to work with homogeneous data sets, obtained with the same instrument and analysed in the same way; in subtracting them one expects that many of the systematic errors will cancel. For that reason I shall limit the subject of my discussion to the data collected from 1986 to 1990 at the Big Bear Solar Observatory (BBSO), presented by Libbrecht \& Woodard (1990) and Woodard \& Libbrecht (1993a, 1993b). However, one must be warned of a particularly insidious problem apparently associated with the filtering by the observing window function, which has most power at $1 \mathrm{~d}^{-1}=11.6 \mu \mathrm{Hz}$, with the signal from modes of adjacent degree whose multiplet frequencies differ by a similar amount. Backmann et al. (1993) report a contribution to their splitting data which might arise from such filtering. Unfortunately, it is not invariant in time, and even changes sign. It is also unfortunate that, as Schou (1993) has pointed out, modes of different order that suffer from this coincidence all penetrate to the same depth, to about $r=0.8 R$, so they experience essentially identical averages of $\Omega$, at least if $\Omega$ varies on a length scale greater than the characteristic wavelength of the oscillations. Unlike many other errors, such a systematic error cannot therefore be revealed from the inversions as an obvious inconsistency in the data.

The BBSO rotational splitting data were originally represented by the first three odd coefficients $\alpha_{i}$ in an expansion in Legendre polynomials in $m / \Lambda$, from which inversions, evidently poorly resolved in latitude, have been carried out (Gough et al. 1993). Some variation between the years is evident, particularly in the polar regions, though it is noticeable that much of that occurs near or above a radius of $0.8 R$. The variation has been related to large-scale magnetic-field patterns by Komm et al. (1993). Unfortunately, the measurements are separated by too great a time interval to determine whether any of the features propagate, as is predicted by many dynamo theories. There is really no firm evidence for vertical propagation, for example, which is consistent with the finding of Dziembowski \& Goode (1993) that the angular momenta of spherical shells appear not to vary in time. Furthermore, the spherically averaged angular momentum density $h$ varies with $r$ continuously across the base of the convection zone (Dziembowski \& Goode 1993), despite a near discontinuity in angular velocity Goode \& Dziembowski (1993), in agreement with previous evidence presented by Brown et al. (1989) and earlier anticipations (Gough 1985). It implies that there is probably no substantial net torque between the convection zone and the radiative interior.

A statistical analysis of the BBSO rotational splitting data, represented by the three odd coefficients $\alpha_{i}$, does indicate some significant temporal variation (Gough \& Stark $1993 \mathrm{a}, 1993 \mathrm{~b})$. In particular, certain radial averages $\bar{\Omega}(\theta, t)$ of $\Omega$ appear to have increased 
from 1986 to 1988 near latitude $20^{\circ}$, the location of maximum sunspot activity, and near latitudes $50^{\circ}-60^{\circ}$. A change in $\bar{\Omega}$ in the vicinity of the sunspots is hardly surprising, because it is presumably a region of dynamical activity, though one must be aware that that activity might also have biassed the data. A marked change in $\Omega$ at high latitude is also not unexpected, because the moment of inertia is smaller there. Indeed, it is particularly likely in view of the temporal invariance of $h$, which suggests that transport of angular momentum is principally on spherical surfaces, even in the convection zone. However, it must be borne in mind that the inferences at the pole are less significant because the rotation (considered as a function of latitude) has a lesser influence on the degeneracy splitting. As time progressed beyond 1988, the increase in $\bar{\Omega}$ was found to become significant at all latitudes, except near $30^{\circ}$ where the magnitude of the $\theta$ dependent contribution to $\tilde{\Omega}$ is relatively insensitive to $\alpha_{3}$ and $\alpha_{5}$. In addition, a radial trend emerged, augmenting both the negative mean radial gradient in $\Omega$ at latitudes above about $30^{\circ}$, and the positive gradient near the equator.

In a recent paper, Woodard \& Libbrecht (1993b) have reported an extension of the Legendre expansion of the rotational splitting to six terms, and discuss the variation of $\bar{\Omega}$, now more highly resolved with respect of $\theta$ than previous estimates. They too report the greatest mean angular velocity change to be at high latitudes, near $60^{\circ}$; in the equatorial regions the greater latitudinal resolution revealed a rather more complicated pattern of temporal change than was discernible in the three-term expansion. No evidence of latitudinal propagation of $\bar{\Omega}$, as one might expect from the torsional oscillations of LaBonte \& Howard (1982), was detected. It will be interesting to see what is revealed by full inversions of the data.

\section{Concluding remarks}

I conclude with a few further brief remarks about several phenomena raised at this meeting that await explanation. The first is to point out that the frequency shifts depicted in Figure 1 exhibit a rapid decline in the vicinity of the theoretical chromospheric resonance frequency, which is probably a little above $4 \mathrm{mHz}$. Such a sharp drop was a property of the shifts computed by Gough \& Thompson (see Gough 1990a), and resulted from the fact that the structure of the model atmosphere did not change substantially as the subphotospheric layers varied; the frequency shift of chromospheric modes was therefore very small. However, the resonance was sharp, and just above the resonant frequency the shift continued along the original curve. This behaviour does not seem to agree with the rather smoother decline in the observations. Goldreich et al. (1991), on the other hand, assume that both chromospheric temperature and fibril fields increase towards solar maximum; modes resonating with the chromospheric cavity are influenced more by the chromospheric temperature rise, and therefore suffer a decline in frequency. The decline is gentler than that found by Gough and Thompson, and can be made to fit the observations very well. Once again, the shift returns to being positive at frequencies above resonance. Recently, Jain \& Roberts (1993) have investigated the combined effect of a horizontal chromospheric magnetic field and a chromospheric temperature rise in a simple model. This produces two terms in the polynomial $Q$ in the formula for the frequency shift quoted in Section 4: a linear term from the magnetic field and a cubic term from the temperature change. Thus, as $\nu$ increases, dominance switches from changes in the Lorentz forces to changes in sound speed and hydrostatic structure, and the frequency perturbation declines and becomes negative. However, because both mechanisms take place in the same location, there is no return to positive values at higher frequency.

Whilst on the subject of the frequency shifts, I wish to draw attention to the hint in 
Figure 1 of an oscillatory deviation of the observations from the smooth curve. This feature was pointed out by Goldreich et al. (1991), and is probably the signature of a changing property of the solar structure that is localized in radius and situated at an acoustical depth of about $(2 P)^{-1}$ beneath the photosphere (more strictly speaking, beneath the upper turning points of the modes), where $P$ is the "period" (with respect to $\nu$ ) of the oscillation (Gough 1990a). It is evident from the figure that $P \simeq 680 \mu \mathrm{Hz}$, which corresponds to a region of variation situated about $0.03 R$ beneath the photosphere, located in the He II ionization zone where about 60 percent of the helium is completely ionized. This immediately raises the issue of the influence of the magnetic field both on the thermodynamic properties of the gas, principally through its influence on the adiabatic exponent $\gamma$ via ionization which modifies the variation of sound speed in the ionization zone, and the hydrostatic stratification through its influence on the adiabatic gradient and on the balance of forces, which both modifies the ionization zone and moves it relative to the upper turning points of the acoustic modes. However, in seeking an explanation of the phenomenon, one must be aware that the oscillation might just be a product of the manner in which the data were binned. I must also point out that although most of the variation in the frequencies of the low-degree modes, illustrated in Figures 1 and 2, can be accounted for by the surface phenomena responsible for the shifts observed by Libbrecht and Woodard, it is by no means clear that the whole of the variation can be so explained. If there remains an additional contribution, that would be indicative of a much more deeply seated variation associated with the solar cycle, possibly in the solar core.

Finally, I return to Kuhn's interesting discussion at this meeting. His numerical convection experiments were very instructive, but were not, if I understood correctly, carried out consistently over the entire convection zone. To do so would still be a daunting task, which is why stellar modellers often continue to revert to mixing-length models. And I conclude by doing so myself. In particular, I revisit the point raised by Kuhn that convection transports heat perturbations vertically more readily than it does horizontally, which is why a magnetically induced thermal anomaly starting out at the base of the convection zone is not severely washed out by the time it reaches the photosphere. How is that explained? Let us estimate the fate of a lateral temperature discontinuity. I shall at first temporarily assume that the usual small-eddy-size approximation is valid, even though that is certainly not the case in the lower regions of the zone. Nonetheless, if it were, then the anomaly would rise through the convection zone with the rms convective velocity $w$ (Gough 1981), and suffer lateral diffusion that tends to smooth out the discontinuity. Assuming that, as the anomaly rises, the lateral turbulent diffusion coefficient $\kappa=\beta w l$, where $l$ is the mixing length and $\beta$ is a dimensionless correlation parameter of order unity, varies slowly compared with the evolution of the discontinuity, one can estimate the scale width of the smoothed discontinuity (at its mid-point) at time $t$ to be $\left(\pi \int \kappa d t\right)^{1 / 2}$. In this simplified discussion, I ignore the dynamical influence of the lateral temperature variation on the convection. Since the disturbance is travelling upwards with speed $w$, the scale width at the photosphere is approximately $\sigma=\left(\pi \int \kappa w^{-1} d r\right)^{\frac{1}{2}}$, where the integral extends from the bottom to the top of the convection zone. If one now sets $l=\alpha H$, where $H$ is the local pressure scale height and $\alpha$ is another constant of order unity, and adopts the approximation $H \simeq 0.4 z$, where $z=R-r$ is depth beneath the photosphere, one readily deduces that $\sigma \simeq(0.2 \pi \alpha \beta)^{\frac{1}{2}} d$, where $d \simeq 0.3 R$ is the depth of the convection zone. Setting $\alpha \simeq 2$ and $\beta \simeq \frac{1}{2}$, say, yields $\sigma \simeq 0.24 R$, which subtends an angle of approximately $14^{\circ}$ on the solar surface (about the centre of the Sun). Of course this estimate exaggerates the diffusion in any particular realization, because in reality the eddies deep in the convection zone are larger than the characteristic lateral 
scale of variation of the anomaly, so part of the influence of convection would be more akin to laminar advection than diffusion. In that case an initial near discontinuity would emerge at the surface on a scale less than $14^{\circ}$, but would be moved and distorted within that characteristic angle. If the convection were truly random, the $14^{\circ}$ would then represent the scale of a frequency (probability) distribution of realizations. In any case, it is evident from this estimate that a thermal (density) anomaly whose lateral scale exceeds $14^{\circ}$ would not be obliterated as it rises to the photosphere. I must emphasize that this estimate relates only to density anomalies, which produce buoyancy and contribute to the dynamics of the convection. A passive scalar diffuses both horizontally and vertically, and would suffer rather more smoothing. Evidently, one of the tasks of the helioseismologists is to test Kuhn's ideas by analysing the even splitting coefficients in search for corrugations in the base of the convection zone. I tried that once using the early data provided by Libbrecht \& Woodard (1990), but was unsuccessful. Perhaps the new data will bear fruit.

\section{REFERENCES}

Bachmann, K. T. \& Brown, T. M. 1993 P-mode frequency variation in relation to global solar activity. Astrophys. J. Lett. 411, L45-L48.

BachmanN, K. T., Schou, J. \& Brown, T. M. 1993 Observations of intermediate-degree solar oscillations: 1989 April-June. Astrophys. J. 412, 870-879.

Balmforth, N. J., Gough, D. O. \& Merryfield, W. J. 1993 Structural changes of the Sun during the solar cycle. Mon. Not. R. astr. Soc., submitted.

Brown, T. M. 1987 Ground-based observations relating to the global state of the Sun. Solar Radiative Output Variation (ed. P. Foukal). pp. 176-188. NCAR, Boulder, CO, USA.

Brown, T. M., Christensen-Dalsgaard, J., Dziembowski, W. A., Goode, P. R., Gough, D. O. \& MORRow, C. A. 1989 Inferring the Sun's internal angular velocity from observed p-mode frequency splittings. Astrophys. J. 343, 526-546.

CAMpbell, W. R. \& RoBerts, B. 1989 The influence of a chromospheric magnetic field on the solar $\mathrm{p}$ and $\mathrm{f}$ modes. Astrophys. J. 338, 538-556.

DÄPPEN, W. 1983 Hydrostatic reaction of the Sun to local disturbances. Astron. Astrophys. 124, 11-22.

Delache, Ph., Gavryusev, V., Gavryuseva, E., laclare, F. Régulo, C. \& Roca CoRTÉs, T. 1993 Time correlation between solar structural parameters: p-mode frequencies, radius, and neutrino flux. Astrophys. J. 407, 801-805.

Duvall JR, T. L., Dziembowski, W. A., Goode, P. R., Gough, D. O., Harvey, J. W. \& LEIBACHER, J. W. 1984 The internal rotation of the Sun. Nature 310, 22-25.

Duvall JR, T. L., Harvey, J. W. \& Pomerantz, M. A. 1986 Latitude and depth variation of the solar rotation. Nature 321, 500-501.

DzIembowski, W. A. \& Goode, P. 1993 The Sun's internal angular velocity from seismology. GONG 1992: Seismic Investigation of the Sun and Stars (ed. T. M. Brown). Astr. Soc. Pac. Conf. Ser. Vol. 42, pp. 225-227.

Elsworth, Y., Howe, R., Isaak, G. R., McLeod, C. P. \& New, R. 1990 Variation of low-order acoustic solar oscillations over the solar cycle. Nature 345, 322-324.

Evans, D. J. \& Roberts, B. 1990 The influence of a chromospheric magnetic field on the solar p and $f$ modes, II: uniform chromospheric field Astrophys. J. 356, 704-719.

Evans, D. J. \& RoBerTs, B. 1991 The sensitivity of chromospherically induced p- and f-mode frequency shifts to the height of the magnetic canopy. Astrophys. J. 371, 387-395.

Evans, D. J. \& Roberts, B. 1992 Interpretation of solar-cycle variability in high-degree p-mode frequencies. Nature 355, 230-232.

Goldreich, P. Murray, N. Willette, G. \& Kumar, P. 1991 Implications of solar p-mode frequency shifts. Astrophys. J. 370, 752-762. 
Goode, P. R. \& Dziembowski, W. A. 1990 Solar-cycle dependence of the Sun's deep internal rotation shown by helioseismology. Nature 349, 223-225.

Goode, P. R. \& Dziembowski, W. A. 1993 The Sun's internal rotation during and after the 1986 activity minimum. GONG 1992: Seismic investigation of the Sun and Stars (ed. T. M. Brown). Astr. Soc. Pac. Conf. Ser. vol. 42, 217-219.

Goode, P. R., Dziembowski, W. A. Korzennik, S. G. \& Rhodes JR, E. J. 1991 What we know about the Sun's internal rotation from solar oscillations. Astrophys. J. 367, 649-657.

Goode, P. R. \& KuHN, J. R. 1990 Consistency in trends in helioseismic data and photospheric temperature data through the solar cycle. Astrophys. J. 356, 310-314.

Gough, D. O. 1981 On the seat of the solar cycle. In Variations of the Solar Constant (ed. S. Sofia). NASA Conf. Publ. 2191, 185-206.

Gough, D. O. 1985 Immediate and long-term prospects for helioseismology. Adv. Sp. Res. 4, 85-102.

GovGH, D. O. 1990 Shaky clues to solar activity. Nature 345, 768-769.

Gough, D. O. 1990 Comments on helioseismic inference. In Progress in Seismology of the Sun and Stars (ed. Y. Osaki \& H. Shibahashi). pp. 283-318. Springer, Heidelberg.

Gough, D. O., Kosovichev, A.G., Sekil, T., Libbrecht, K. G. \& Woodard, M. F. 1993 Seismic evidence of modulation of the structure and angular velocity of the Sun associated with the solar cycle. In Inside the Stars (ed. W. W. Weiss \& A. Baglin). Astr. Soc. Pac. Conf. Ser. Vol. 40, 93-96.

Gough, D. O. \& Novotny, E. 1993 On the asteroseismic calibration of a solar-type star. In Inside the Stars (ed. W. W. Weiss \& A. Baglin). Publ. Astr. Soc. Pac. Conf. Ser. Vol. 40, pp. 550-553.

Gough, D. O. \& Stark, P. B. 1993a Are the 1986-1988 changes on solar free-oscillation frequency splitting significant? Astrophys. J. 415, 376-382.

Gough, D. O. \& STARK, P.B. 1993b The significance of changes in solar free-oscillation splitting from 1986 to 1990. In GONG 1992: Seismic Investigation of the Sun and Stars (ed. T. M. Brown). Astr. Soc. Pac. Conf. Ser. Vol. 42, 221-224.

Gough, D. O. \& Thompson, M. J. 1988 Magnetic perturbations to stellar oscillation eigenfrequencies. In Advances in Helio- and Asteroseismology (ed. J. Christensen-Dalsgaard \& S. Frandsen). pp. 175-180. Reidel, Dordrecht.

JAIN, R. \& RoBerTs, B. 1993 Do p-mode frequency shifts suggest a hotter chromosphere at solar maximum? Astrophys. J. 414, 898-907.

Komm, R. W., Harvey, J. W. \& Howard, R. F. 1993 Torsional oscillations and internal rotation. In GONG 1992: Seismic Investigation of the Sun and Stars (ed. T. M. Brown). Astr. Soc. Pac. Conf. Ser. Vol. 42, pp. 269-272.

KUHN, J. R. 1988a Radial and temporal structure of the internal solar asphericity. In Seismology of the Sun and Sun-Like Stars (ed. V. Domingo \& E. J. Rolfe). pp. 87-90 ESA SP-286, Noordwijk.

KuHN, J. R. 1988b Helioseismological splitting measurements and the nonspherical solar temperature structure. Astrophys. J. Lett. 331, L131-L134.

Kunn, J. R. 1989a Helioseismic observations of the solar cycle. Astrophys. J. Lett. 339, L45-L47.

KuHn, J. R. 1989b Calculating the internal solar asphericity from frequency splitting measurements. Solar Phys. 123, 1-5.

KuHN, J. R. 1990 Measuring solar structure variations from helioseismic and photometric observations. In Seismology of the Sun and Stars (ed. Y. Osaki and H. Shibahashi). pp. 157-162. Springer, Heidelberg.

KuhN, J. R., LiBbrecht, K. G \& Dicke, R. H. 1985 Observations of a solar latitudedependent limb brightness variation. Astrophys. J. 290 758-764 (erratum 319, 1010 (1987)

Kuhn, J. R., Libbrecht, K. G. \& Dicke, R. H. 1988 The surface temperature of the Sun and changes in the solar constant. Science 242, 908-911.

Kyle, H. L., Hoyt, D. V. \& Hickey, J. R. 1994 A review of the Nimbus-7 ERB solar dataset. 
Solar Phys., in press.

LaBonte, B. \& Howard, R. F. 1982 Are the high-latitude torsional oscillations of the Sun real? Solar Phys. 80, 373-378.

LiBbrecht, K. G. \& WOODARD, M. F. 1990 Solar-cycle effects on solar oscillation frequencies. Nature 345, 779-782.

PARKer, E. N. 1987 Magnetic activity complexes, thermal relaxation oscillations, and the dynamics of the azimuthal magnetic field of a star. In The Internal Solar Angular Velocity (ed. B. R. Durney \& S. Sofia). pp. 289-301. Reidel, Dordrecht.

Schou, J. 1991 An inversion for the rotation rate of the solar interior. In Challenges to Theories of the Structure of Moderate-Mass Stars (ed. D. O. Gough \& J. Toomre). pp. 81-86. Springer, Heidelberg.

SCHOU, J. 1993 On the analysis of helioseismic data. Ph.D thesis, University of Aarhus.

SPRUIT, H. 1990 Theory of luminosity and radius variations. In The Sun in Time (ed. C.P. Sonett, M.S. Giampapa \& M.S. Matthews). pp. 118-158. Univ. Arizona Press, Tucson, AZ, USA.

ULRICH, R. K. 1986 Determination of stellar ages from asteroseismology. Astrophys. J. Lett. 306, L37-L40

Willson, R.C. \& Hudson, H.S. 1988 Solar luminosity variations in solar cycle 21. Nature, 332, 810-812

Woodard, M. F. \& LibBrecht, K. G. 1993a Solar activity and oscillation frequency splittings. Astrophys. J. Lett. 402, L77-L80

WoOdARD, M. F. \& LibBrechT, K. G. 1993b Observations of time variation in the Sun's rotation. Science 260, 1178-1181

Woodard, M. F., Kuhn, J. R., Murray, N. \& Libbrecht, K. B. 1991 Short-term changes in solar oscillation frequencies and solar activity. Astrophys. J. Lett. 373, L81-L84

Wright, A. N. \& Thompson, M. J. 1992 On the effects of chromospheric magnetic perturbations on solar oscillation frequencies. Astron. Astrophys. 264, 701-707. 